\title{
Targeted systemic therapies for primary brain tumors
}

The diagnosis and treatment of malignant brain tumors have been a challenge in the past decades. However, great strides have been made recently in the field of neuro-oncology with advances in diagnostic tools, neurosurgical techniques, radiotherapeutic applications, development of potent chemotherapeutic agents, immuno-oncologic agents, dendritic cell vaccines and oncolytic viral therapies. In addition, new biologic modifiers such as antiangiogenic agents and molecular targeted therapies are changing the paradigm of diagnostic and therapeutic approaches based on their molecular genetic profiling. In 2016, for the first time, new WHO Classification Tumors of the Central Nervous System incorporated molecular parameters to histology to define many brain tumor types, thus, opening a new era for molecular based diagnosis and treatment. For example, glioblastoma is now subclassified based on MGMT methylation and isocitrate dehydrogenase (IDH) mutation status. Oligodendroglioma is now diagnosed based on $1 \mathrm{IDH}$ mutation and $1 \mathrm{p} 19 \mathrm{q}$ co-deletion status and even if the tumor appears to resemble oligodendroglioma morphologically, but does not express IDH mutation and 1p19q co-deletion, then, it cannot be considered as oligodendroglioma but as astrocytoma.

The advent of molecular profiling including next generation sequencing with advances in understanding molecular genetics opened a new door for the treatment of malignant gliomas and tumors that were previously thought to be not treatable or resistant to conventional chemotherapeutic agents. High grade gliomas including glioblastoma have a poor prognosis with a median survival of $14-18$ months with 5 -year overall survival of $<10 \%$. Traditionally, the treatment of malignant gliomas including glioblastoma has been surgery and radiation therapy, however, the overall survival remained poor. The role of chemotherapy has been elusive with no clear benefit of improved survival until the introduction of temozolomide for the treatment of glioblastoma which has shown a clear benefit of tumor response and survival extending 5 years and beyond in a small group of long-term survivors, particularly, with MGMT methylation. There are other potential molecular targets including vascular endothelial growth factor (VEGF), epidermal growth factor receptor (EGFR), programmed cell death protein 1 (PD-1)/programmed death-ligand 1 (PD-L1) and multi tyrosine kinases of many different pathways for therapeutic implications. Diffuse midline glioma is highly aggressive glioma affecting children and young adults that has a very poor prognosis especially with H3K27M mutation. However, ONC201, a highly selective antagonist of dopamine receptor D2 (DRD2) and ClpP agonist, was found to be highly effective in a small trial for H3K27M mutant gliomas and if ongoing pivotal trial confirms the benefit of this drug, it could become a drug of choice for this indication. Other tumors such as meningiomas, craniopharyngiomas and pituitary tumors that were conventionally known to have no clear therapeutic options except surgery and radiation therapy are now found to have some interesting novel markers that could make them attractive for molecular targeted therapies such as VEGF, platelet-derived growth factor receptor (PDGFR), mammalian target of rapamycin (mTOR) and BRAF inhibitors.

To that extent, in this $C C O$ focused series of the "Targeted systemic therapies for primary brain tumors", Ali et al. and Wagel et al. eloquently discuss the role of targeted treatment options with stereotactic radiation for recurrent gliomas and targeted therapies in glioblastoma respectively while Kim and Martinez et al. extensively review the role of molecular targeted therapy options in the treatment of meningioma and other skull based tumors such as pituitary tumor and craniopharyngioma, respectively.

In conclusion, advances in medical engineering and nanotechnology that can enable us to identify specific biological markers and potential targets and subsequent development of therapeutic agents with better central nervous system (CNS) penetration and drug delivery system, advances in molecular genetic profiling and alteration, better understanding of cancer stem cell and inhibition and development of immuno-oncologic therapies, oncolytic viral therapies and vaccines are opening new opportunities for better targeted therapeutic options for the treatment of primary brain tumors based on their molecular characteristics that hopefully would improve the overall management and the outcome of the primary brain tumors.

\section{Acknowledgments}

I am deeply grateful to the authors for their outstanding contributions, CCO senior editor, Teresa Lin and CCO for their generous support with this series.

Funding: None. 


\section{Footnote}

Provenance and Peer Review: This article was commissioned by the editorial office, Chinese Clinical Oncology for the series "Targeted systemic therapies for primary and metastatic brain tumors". The article did not undergo external peer review.

Conflicts of Interest: The author has completed the ICMJE uniform disclosure form (available at http://dx.doi.org/10.21037/ cco-2020-mbt-05). The series "Targeted systemic therapies for primary and metastatic brain tumors" was commissioned by the editorial office without any funding or sponsorship. The author has no other conflicts of interest to declare.

Ethical Statement: The author is accountable for all aspects of the work in ensuring that questions related to the accuracy or integrity of any part of the work are appropriately investigated and resolved.

Open Access Statement: This is an Open Access article distributed in accordance with the Creative Commons AttributionNonCommercial-NoDerivs 4.0 International License (CC BY-NC-ND 4.0), which permits the non-commercial replication and distribution of the article with the strict proviso that no changes or edits are made and the original work is properly cited (including links to both the formal publication through the relevant DOI and the license). See: https://creativecommons.org/licenses/by-nc$\mathrm{nd} / 4.0 /$.

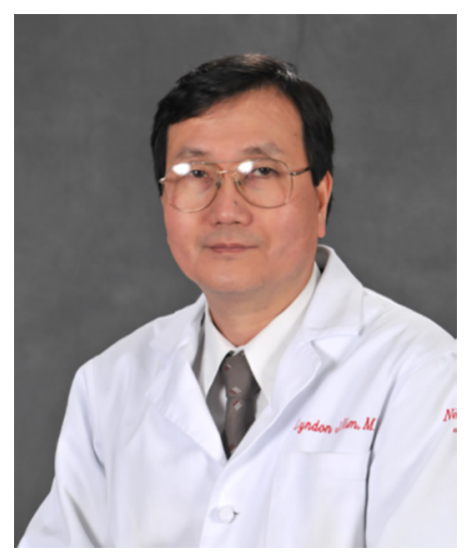

Lyndon Kim

Lyndon Kim, MD

Division of Neuro-Oncology, Department of Neurology, Medicine and Neurosurgery, Mount Sinai Hospital, Icahn School of Medicine at Mount Sinai, New York, NY, USA.

(Email: neurooncology@netscape.net; lyndon.kim@mssm.edu)

Submitted Dec 14, 2020. Accepted for publication Dec 30, 2020.

doi: $10.21037 /$ cco-2020-mbt-05

View this article at: http://dx.doi.org/10.21037/cco-2020-mbt-05

Cite this article as: Kim L. Targeted systemic therapies for primary brain tumors. Chin Clin Oncol 2020;9(6):73. doi: 10.21037/cco-2020-mbt-05 